\title{
Fractal Sets as Final Coalgebras Obtained by Completing an Initial Algebra
}

\author{
Lawrence S. Moss* Jayampathy Ratnayake, and Robert Rose \\ Mathematics Department, Indiana University, Bloomington, IN 47405 USA
}

\begin{abstract}
This paper is a contribution to the presentation of fractal sets in terms of final coalgebras. The first result on this topic was Freyd's Theorem: the unit interval $[0,1]$ is the final coalgebra of a functor $X \mapsto X \oplus X$ on the category of bipointed sets. Leinster [L] offers a sweeping generalization of this result. He is able to represent many of what would be intuitively called self-similar spaces using (a) bimodules (also called profunctors or distributors), (b) an examination of non-degeneracy conditions on functors of various sorts; (c) a construction of final coalgebras for the types of functors of interest using a notion of resolution. In addition to the characterization of fractals sets as sets, his seminal paper also characterizes them as topological spaces.

Our major contribution is to suggest that in many cases of interest, point (c) above on resolutions is not needed in the construction of final coalgebras. Instead, one may obtain a number of spaces of interest as the Cauchy completion of an initial algebra, and this initial algebra is the set of points in a colimit of an $\omega$-sequence of finite metric spaces. This generalizes Hutchinson's characterization of fractal attractors in $[\mathrm{H}]$ as closures of the orbits of the critical points. In addition to simplifying the overall machinery, it also presents a metric space which is "computationally related" to the overall fractal. For example, when applied to Freyd's construction, our method yields the metric space of dyadic rational numbers in $[0,1]$.
\end{abstract}

Our second contribution is not completed at this time, but it is a set of results on metric space characterizations of final coalgebras. This point was raised as an open issue in Hasuo, Jacobs, and Niqui [HJ], and our interest in quotient metrics comes from [HJN]. So in terms of (a)-(c) above, our work develops (a) and (b) in metric settings while dropping (c).

\section{Introduction}

As our abstract above indicates, this paper is largely a kind of "marginal note" to Leinster's paper on self-similar spaces obtained as final coalgebras of a certain sort. Our 4-page TACL abstract cannot even hope to present all of Leinster's definitions and central results, and so we must refer the reader to $[\mathrm{L}$ for all of the background. In fact, we are going to take the unusual step of not even presenting all of our own definitions and results, since a reader unfamiliar would not be able to follow a brief presentation, and a reader who has studied [ $\mathrm{L}$ and/or [HJN] would likely see our general formulations. In addition to the "marginal note" on using completions of initial algebras, we are interested in metric-space versions of non-degeneracy conditions and recognition theorems from $[\mathrm{L}$. Our contributions here involve borrowings from metric geometry (see $[\overline{\mathrm{BH}}]$ ). And so our remarks in this abstract are mainly hints as well.

Now that we have said mentioned that this abstract is not a decent presentation of our results, let alone of Leinster $[\mathrm{L}$, we should say what we are presenting. We are going to give a a concrete example of how our constructions work, aimed mainly at the uninitiated reader. At times we shall of course mention connections to other papers, especially Leinster $[\mathrm{L}$.

*This work was partially supported by a grant from the Simons Foundation (\#245591 to Lawrence Moss). 


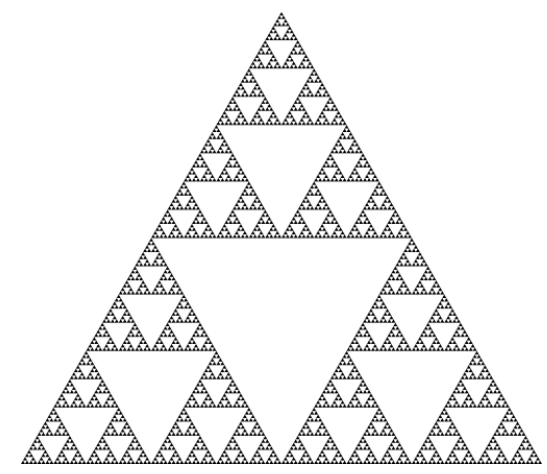

Figure 1: The Sierpiński gasket $\mathbb{S} \subseteq \mathbb{R}^{2}$. The vertices are top $=(1 / 2, \sqrt{3} / 2)$, left $=(0,0)$, and right $=(1,0)$.

\section{The Sierpiński Gasket}

Consider the maps $\sigma_{a}, \sigma_{b}, \sigma_{c}: \mathbb{R}^{2} \rightarrow \mathbb{R}^{2}$ given by

$$
\begin{aligned}
& \sigma_{a}(x, y)=(x / 2, y / 2)+(1 / 4, \sqrt{3} / 4) \\
& \sigma_{b}(x, y)=(x / 2, y / 2) \\
& \sigma_{c}(x, y)=(x / 2, y / 2)+(1 / 2,0)
\end{aligned}
$$

These maps $\sigma_{i}$ extend to subsets of $\mathbb{R}^{2}$ by taking images. The Sierpiński gasket is the unique non-empty compact subset $\mathbb{S} \subseteq \mathbb{R}^{2}$ such that

$$
\mathbb{S}=\sigma_{a}(\mathbb{S}) \cup \sigma_{b}(\mathbb{S}) \cup \sigma_{c}(\mathbb{S})
$$

$\mathbb{S}$ is shown in Figure 1.

Let $M=\{a, b, c\}$. We use the letter $m$ as a variable over $M$ in the sequel. Let $M^{*}$ be the set of finite words from $M$. We define triangles $t r_{w}$ for $w \in M^{*}$ by recursion:

$$
\begin{aligned}
t r_{\varepsilon} & =\text { the triangle with vertices top }=(1 / 2, \sqrt{3} / 2), \text { left }=(0,0), \text { and right }=(1,0) \\
t r_{m w} & =\sigma_{m}\left(t r_{w}\right)
\end{aligned}
$$

Let

$$
R_{n}=\bigcup\left\{t r_{w}: w \text { is a word of length } n\right\}
$$

Also, for an infinite sequence $\alpha=\alpha_{0} \alpha_{1} \cdots \alpha_{n} \cdots \in M^{\omega}$, let

$$
p_{\alpha}=\text { the unique point in } \bigcap_{n} t r_{\alpha_{0} \alpha_{1} \cdots \alpha_{n}}
$$

That is, $\bigcap_{n} t r_{\alpha_{0} \alpha_{1} \cdots \alpha_{n}}$ is an intersection of a family of non-empty compact sets, so it is a singleton.

Proposition 2.1 (A very special case of Hutchinson $[\mathrm{H}]$ ). $\mathbb{S}$ has the following characterizations:

$$
\mathbb{S}=\bigcap_{n} R_{n}=\left\{p_{\alpha}: \alpha \in M^{\omega}\right\}=\text { the closure of } \bigcup_{w \in M^{*}}\left\{x: x \text { is a vertex of } t_{w}\right\} .
$$


Remark Different sequences in $M^{\omega}$ might well be associated with the same point via the operation $p$. For example, let $\alpha=$ baccc $\cdots$, and let $\beta=$ bcaaa $\cdots$. Then $p_{\alpha}=p_{\beta}=(3 / 8, \sqrt{3} / 8)$.

Remark Proposition 2.1 gives different ways to think about $\mathbb{S}$. Writing $\mathbb{S}=\left\{p_{\alpha}: \alpha \in M^{\omega}\right\}$ connects $\mathbb{S}$ with resolutions as in Leinster $\underline{\mathrm{L}}$. The final way is closest to what we do in this paper.

\subsection{Tripointed Sets}

Definition A tripointed set is a set $X$ together with distinguished elements top, left, and right. $X$ is non-degenerate if the distinguished elements are distinct. We let Tri be the category of non-degenerate tripointed sets, taking as morphisms the functions respecting the distinguished points. The initial object $I$ of Tri is $\{$ top, left, right $\}$.

The functor $X \mapsto M \times X$ on Set is defined as usual.

The functor $X \mapsto M \otimes X$ on Tri $\quad$ We define $\sim$ on $M \times X$ to be the relation

$$
\{((a, \text { left }),(b, \text { top })),((a, \text { right }),(c, \text { top })),((b, \text { right }),(c, \text { left }))\} .
$$

We take $M \otimes X$ to be the quotient of $M \times X$ by the smallest equivalence relation including $\sim$. We write $m \otimes x$ instead of the equivalence class $[(m, x)]$. So we have equalities

$$
a \otimes \text { left }=b \otimes \text { top } \quad a \otimes \text { right }=c \otimes \text { top } \quad b \otimes \text { right }=c \otimes \text { left }
$$

We call these points the connection points of $M \otimes X$.

Returning to our tripointed set $M \otimes X$, we now see that this set is a tripointed space with top $_{M \otimes X}=a \otimes$ top, left $M \otimes X b \otimes$ left, and right ${ }_{M \otimes X}=c \otimes$ right. If $X$ is a ND tripointed set, so is $M \otimes X$. The operation $X \mapsto M \otimes X$ extends to a functor Tri $\rightarrow$ Tri. If $f: X \rightarrow Y$ is a morphism of tripointed sets, then $M \otimes f$ preserves the connection points.

The reader familiar with $[\mathrm{L}$. will recall that everything we have done so far is contained in Example 10.10. In particular the requirement that top, left, and right be distinct is a special case of non-degeneracy.

\section{$2.2 \quad$ Tripointed Metric Spaces}

The functor $X \mapsto M \times X$ on MS MS is the category of metric spaces. (We do not assume that all distances are bounded by 1.) The morphisms in MS are the non-expanding maps of metric spaces. Then $X \mapsto M \times X$ extends to an endofunctor on MS in the following way. We take

$$
d((m, x),(n, y))= \begin{cases}\frac{1}{2} d(x, y) & \text { if } m=n \\ 1 & \text { if } m \neq n\end{cases}
$$

If $f: X \rightarrow Y$ is a morphism in MS, then so is $M \times f: M \times X \rightarrow M \times Y$. It is easy to show that if $X$ is complete, then $M \times X$ is also complete. $X \mapsto M \times X$ is contracting: for $f, g: X \rightarrow Y$, $d(M \times f, M \times g) \leq \frac{1}{2} d(f, g)$. 
The functor $X \mapsto M \otimes X$ on TriMS An object of TriMS is a metric space with three distinguished points top, left, and right which are required to be of pairwise distance 1 .

Example 2.2. The initial object $I$ of TriMS is the same set $I=\{$ top, left, right $\}$ with pairwise distances 1 . Another example is $\mathbb{R}^{2}$ with the usual metric, taking top $=(\sqrt{3} / 2,1)$, left $=(0,0)$, and right $=(1,0)$.

We turn $X \mapsto M \otimes X$ to an endofunctor on Trims by taking the set $M \otimes X$ and using the quotient metric via the equivalence relation $\sim$.

Lemma 2.3. For all $x, y \in X$, distances in $M \otimes X$ are calculated as follows:

$$
\begin{aligned}
& d(a \otimes x, a \otimes y)=\frac{1}{2} d(x, y) \\
& d(a \otimes x, b \otimes y)=\min \left(\frac{1}{2}(d(a, \text { left })+d(\text { top }, y)), \frac{1}{2}(d(x, \text { right })+1+d(\text { left }, y))\right)
\end{aligned}
$$

and similarly for the other distance calculations.

Lemma 2.3 implies that $M \otimes X$ is non-degenerate whenever $X$ is.

There is a quotient natural transformation $q: M \times X \rightarrow M \otimes X$.

\subsection{Isometric Embeddings and the Initial Chain}

A TriMS morphism $\eta: X \rightarrow Y$ is an isometric embedding if it preserves all distances.

Proposition 2.4. Concerning isometric embeddings:

1. The unique map $\eta: I \rightarrow M \otimes I$ is an isometric embedding, where $I$ is the initial object of Trims.

2. If $\eta: X \rightarrow Y$ is an isometric embedding, so is $M \otimes \eta: M \otimes X \rightarrow M \otimes Y$.

As a result, we have a chain of isometric embeddings

$$
I \stackrel{\eta}{\longrightarrow} M \otimes I \stackrel{M \otimes \eta}{\longrightarrow} M \otimes M \otimes I \stackrel{M \otimes M \otimes \eta}{\longrightarrow} \cdots
$$

In this situation, the colimit exists and is preserved by the functor $M \otimes-$. We write $G$ for the colimit space. By Adámek's Theorem [A], $G$ carries the structure of an initial algebra $\eta: M \otimes G \rightarrow G$. In particular, $\eta$ is invertible.

We let TriCMS be the tripointed metric spaces which are complete: every Cauchy sequence has a limit. Every metric space is isometrically embedded in its Cauchy completion, and so we have a Cauchy completion functor $C:$ TriMS $\rightarrow$ TriCMS.

Proposition 2.5. The TriMS-endofunctors $X \rightarrow C(M \otimes X)$ and $X \rightarrow M \otimes C(X)$ are naturally isomorphic.

Proof. We check that for all tripointed metric spaces $X, C(M \otimes X)=M \otimes C(X)$. Consider

$$
M \otimes C(X) \stackrel{f}{\longrightarrow} C(M \otimes X) \stackrel{g}{\longrightarrow} M \otimes C(X)
$$

given by

$$
\begin{array}{ll}
f\left(m \otimes\left(x_{0}, x_{1}, \ldots, x_{n}\right)\right) & =\left(m \otimes x_{0}, m \otimes x_{1}, \ldots,\right) \\
g\left(\left(m_{i} \otimes x_{i}\right)_{i}\right) & =m^{*} \otimes\left(x_{j_{1}}, x_{j_{2}}, \ldots,\right)
\end{array}
$$


In the definition of $g$, for each sequence of points in $M \otimes X$, some $m \in M$ must occur infinitely many times as the first coordinate $m_{i}$, since $M$ is finite. Let $m^{*}$ be "first" in some pre-chosen order on $M$. Let $\left(x_{j_{i}}\right)_{i}$ be the subsequence of $\left(x_{i}\right)_{i}$ such that the corresponding first coordinates are all $m^{*}$.

One checks that $f$ and $g$ are inverses (modulo equivalence of Cauchy sequences), that they are non-distance expanding (hence they are isometries), that they preserve the distinguished points, and that they are the components of natural transformations. All of these verifications are elementary.

We have seen the initial algebra $(G, \eta: M \otimes G \rightarrow G)$ of $X \rightarrow M \otimes X$ on Trims. We have also seen the Cauchy completion functor $C$. Let $S=C(G)$. Since $C$ is a functor, we have an isomorphism $C \eta: M \otimes S \rightarrow S$. Our central result implies that $(C \eta)^{-1}: S \rightarrow M \otimes S$ is a final coalgebra for $X \rightarrow M \otimes X$ on Tri.

Theorem 2.6. The final coalgebra of the functor $M \otimes X$ on tripointed sets is the completion of the initial algebra. Moreover, the Sierpiński gasket is bi-Lipschitz homeomorphic to this final coalgebra.

These results are new. The main content of the paper is a general theory of why this is so, building on, and sometimes varying, notions from Leinster $[\mathrm{L}$. For example, A pointed combinatorial specification is a triple $(\mathbb{A}, M$, pt) if $(\mathbb{A}, M)$ is a combinatorial specification, pt is an object of $\mathbb{A}$, and if (1) hom $(\mathrm{pt}, \mathrm{pt})=\left\{\mathrm{id}_{\mathrm{pt}}\right\} ;(2) M(\mathrm{pt}, \mathrm{pt})=1$; and (3) For $a \neq \mathrm{pt}$, $M(a, \mathrm{pt})=0$. These requirements and the Yoneda lemma imply that $\operatorname{Hom}(\mathrm{pt}, a): \mathbb{A} \rightarrow$ Set is an initial non-degenerate functor, and this is how we start the chain (2) of isometric embeddings.

\section{References}

[A] J. Adámek, Free algebras and automata realizations in the language of categories, Comment. Math. Univ. Carolinae 14 (1974), 589-602.

[AMM] J. Adámek, S. Milius and L. S. Moss, Initial algebras and final coalgebras: a survey, ms. 2011.

[BH] Bridson, Martin R. and André Haefliger, Metric spaces of non-positive curvature. Springer-Verlag, Berlin, 1999.

[F] P. Freyd, Real Coalgebra, post on the Categories mailing list, December 22, 1999, available at www.mta.ca/ cat-dist

[HJN] I. Hasuo, B. Jacobs, and M. Niqui, Coalgebraic Representation Theory of Fractals (Extended Abstract), Proc. Mathematical Foundations of Programming Semantics (MFPS XXVI), Electr. Notes Comp. Sci. 265:351-368, 2010.

[H] John Hutchinson, Fractals and Self-Similarity, Indiana Univ. Math. J. 30 No. 5 (1981), 713747.

[L] T. Leinster, A general theory of self-similarity, Advances in Mathematics 226 (2011), 29353017. 